\title{
BRANCH POINTS OF SOLUTIONS OF EQUATIONS IN BANACH SPACE $\left({ }^{1}\right)$
}

\author{
BY \\ JANE CRONIN
}

1. Introduction. F. Riesz $[13]\left({ }^{2}\right), S$. Banach [2] and others have generalized the Fredholm theory of linear integral equations to Banach space. However, except for a short note by R. Caccioppoli [3], nothing has been done regarding the corresponding generalization for the theory of ramifications of solutions arising in the nonlinear integral equation theory as developed by E. Schmidt [17] and also by A. Liapounoff [11]. The object of the work is to fill this gap.

Schmidt considers the problem of determining the local solutions $u(s)$ of an integral equation, the linear part of which is of the form

$$
u(s)-\int_{a}^{b} C(s, t) u(t) d t .
$$

The generalization to Banach space of the case in which this expression, regarded as an operator on $u(s)$, is nonsingular is contained in the work of Hildebrandt and Graves [5]. We consider here the problem of generalizing the case in which the operator is singular, that is, the case in which the operator maps some functions which are not identically zero in to the zero function.

In $\S 2$, known theorems that are to be applied are listed. In $\$ 3$, an equation in Banach space $\mathfrak{X}$ which is a generalization of the integral equation studied by Schmidt is set up. This equation is

$$
(I-C) x+S(y)+T(x, y)=\theta
$$

where $I$ is the identity mapping, $\theta$ is the zero element, $C$ is linear, completely continuous, $S$ is a continuous transformation satisfying a Lipschitz condition, and $T$ is a continuous mapping from $\mathfrak{X} \times \mathfrak{X}$ into $\mathfrak{X}$ with certain $k$ th order properties in $x$, where $k \geqq 2$. (The hypotheses are such that if $T$ is independent of $y$, then the transformation $(I-C)$ is the differential at $\theta$ of the transforma-

Presented to the Society, September 9, 1948; received by the editors January 12, 1949.

(1) Most of this paper is part of a dissertation submitted in partial fulfillment of the requirements for the degree of doctor of philosophy in the University of Michigan. The dissertation was written under the direction of Professor E. H. Rothe of the University of Michigan whose guidance of the work was extremely helpful.

Part of this work was done while the writer was under contract to the Office of Naval Research.

(2) Numbers in brackets refer to the bibliography at the end of the paper. 
tion $I-C+T$.) The problem is to study, in neighborhoods of the given initial solution $x=y=\theta$, the local solutions $x$ of equation (1.1) when $y$ is given.

By applying the Riesz theory of completely continuous operators [13], a multiplicity for the solutions $x$ is defined. In order to define this multiplicity, we use the fact from the Riesz theory that

$$
\mathfrak{X}=\mathfrak{X}_{1}+\mathfrak{X}^{1} \text { (direct sum) }
$$

where $\mathfrak{X}_{1}$ is the null space of the operator $(I-C)$. In [13], it is proved that $\mathfrak{X}_{1}$ is finite-dimensional. Let $E_{1}, E^{1}$ be the projections of $\mathfrak{X}$ into $\mathfrak{X}_{1}, \mathfrak{X}^{1}$ respectively, that is, if $x=x_{1}+x^{1}$ in the direct sum decomposition, then

$$
E_{1}: x \rightarrow x_{1} \quad E^{1}: x \rightarrow x^{1} .
$$

Multiplying equation (1.1) by $E_{1}$ and $E^{1}$, we obtain two equations:

$$
\begin{aligned}
& M_{1}\left(x_{1}, x^{1}, y\right)=\theta, \\
& M^{1}\left(x_{1}, x^{1}, y\right)=\theta
\end{aligned}
$$

in $\mathfrak{X}_{1}$ and $\mathfrak{X}^{1}$ respectively where $x=x_{1}+x^{1}$. Now instead of solving equation (1.1), we study the solutions $x_{1}, x^{1}$ of (1.2) and (1.3) when they are solved simultaneously. It is proved that the linear part of $M^{1}\left(x_{1}, x^{1}, y\right)$ in $x^{1}$ is nonsingular and hence that the implicit function theorem of Hildebrandt and Graves [5] may be applied to obtain a solution $x^{1}=F\left(x_{1}, y\right)$ of equation (1.3). Substituting this expression in equation (1.2), we obtain

$$
M_{1}\left[x_{1}, F\left(x_{1}, y\right), y\right]=\theta .
$$

Thus the problem of studying the solutions $x$ of equation (1.1) is reduced to the study of the solutions $x_{1}$ of equation (1.2') which is an equation in the finite-dimensional Euclidean space $\mathfrak{X}_{1}$. The solutions $x_{1}$ are studied by investigating the topological degree at $\theta$ of the mapping $M_{1}\left[x_{1}, F\left(x_{1}, y\right), y\right]$. We define the multiplicity of the solutions $x$ of the original equation (1.1) to be this topological degree.

We obtain here complete results when the dimension of $\mathfrak{X}_{1}$ is one; if $\mathfrak{X}$ is a Banach space over the complex numbers, it is proved that the multiplicity is $k$, the order of the transformation $T$. If $\mathfrak{X}$ is a Banach space over the real numbers, the multiplicity is 1 or -1 if $k$ is odd and is 0 if $k$ is even. For the case in which the dimension of $\mathfrak{X}_{1}$ is greater than one, some particular examples of the multiplicity are computed. The general problem will be dealt with in a later paper

In $\S 4$, some applications of the theory to differential and integral equations are considered. Finally, in $\$ 5$, the multiplicity defined in $\$ 3$ is connected with the work of Leray and Schauder by showing that if the Leray-Schauder topological degree is defined for the transformation $I-C+T$ in the special case of equation (1.1) 


$$
(I-C+T) x=y,
$$

then the Leray-Schauder degree and the multiplicity we define differ at most by a factor $(-1)$.

2. Some preliminary theorems. For the discussion which follows, we shall need certain known theorems from the theory of Banach spaces. For convenience, we state these theorems in that form in which they will be used here.

Let $\mathfrak{X}$ be a Banach space over the real numbers or the complex numbers $\left({ }^{3}\right)$. In this work, $x, y, z, u, v, w, \cdots$ will be used to denote elements of $\mathfrak{X}$; $\alpha, \beta, \gamma, \cdots$ will denote scalars; and $f, g, h, \cdots$ will denote elements of $\mathfrak{X}^{*}$, the Banach space of linear functionals on $\mathfrak{X}$. Capital letters, $C, I, R, S, T, \cdots$ will be used to denote continuous transformations of $\mathfrak{X}$ or $\mathfrak{X} \times \mathfrak{X}$ into $\mathfrak{X}$. The symbols $\|x\|,\|f\|,\|C\|$ will denote the norms of $x, f$, and $C$ respectively where $C$ is a linear transformation.

First, certain parts of the theory of operators of the form $(I-C)$ where $I$ is the identity and $C$ is linear and completely continuous will be used( $\left.{ }^{4}\right)$. We introduce the notation:

$\mathfrak{X}^{(n)}=(I-C)^{n} \mathfrak{X}$, where $n$ is a positive integer.

$\mathfrak{X}_{n}=$ null space of $(I-C)^{n}$ where $n$ is a positive integer, that is, $\mathfrak{X}_{n}$ is the linear space of elements $z$ in $\mathfrak{X}$ such that $(I-C)^{n} z=\theta$.

THEOREM 2.1. $\mathfrak{X}_{p} \subseteq \mathfrak{X}_{p+8} ; \mathfrak{X}^{(p)} \supseteq \mathfrak{X}^{(p+s)}$ where $p$ and $s$ are arbitrary positive integers.

Theorem 2.2. For each $n, \mathfrak{X}_{n}$ is a finite-dimensional linear space.

THEOREM 2.3. There exists a positive integer $m$, called the index of the transformation $(I-C)$, which is minimal with respect to each of the following two properties:

(a) $\mathfrak{X}_{m}=\mathfrak{X}_{m+n}$ for $n$ any positive integer.

(b) $\mathfrak{X}^{(m)}=\mathfrak{X}^{(m+n)}$ for $n$ any positive integer.

It can be proved that $C^{*}$, the transformation conjugate to $C$ and acting on $\mathfrak{X}^{*}$, is also linear and completely continuous (see [2, pp. 99-101]). Hence the analogs of Theorems 2.1, 2.2, 2.3 hold for $I^{*}-C^{*}$ where $I^{*}$ is the identity transformation on $\mathfrak{X}^{*}$. Moreover, if $\mathfrak{X}_{1}^{*}$ is the null space of $I^{*}-C^{*}$, we have:

Theorem 2.4. Dimension of $\mathfrak{X}_{1}^{*}=$ Dimension of $\mathfrak{X}_{1}$.

Theorem 2.5. There exists a linear, closed subspace $\mathfrak{X}^{1}$ of $\mathfrak{X}$ such that

$$
\mathfrak{X}=\mathfrak{X}_{1} \oplus \mathfrak{X}^{1} \text { (direct sum), }
$$

where $\mathfrak{X}^{1}=\mathfrak{Y}_{1} \oplus \mathfrak{X}^{(m)}$ and $\mathfrak{Y}_{1}$ is such that $\mathfrak{X}_{n}=\mathfrak{X}_{1} \oplus \mathfrak{Y}_{1}$. That is, if $x$ is an element of

(3) See [2].

(4) See [13], [4], and [2]. 
$\mathfrak{X}$, then $x=x_{1}+x^{1}$ where $x_{1}, x^{1}$ are elements of $\mathfrak{X}_{1}$ and $\mathfrak{X}^{1}$ respectively, and the decomposition $x=x_{1}+x^{1}$ is unique.

Now we consider an arbitrary element $x$ of $\mathfrak{X}$. If by the decomposition of Theorem 2.6,

$$
x=x_{1}+x^{1},
$$

then the following transformations, $E_{1}$ and $E^{1}$, may be defined:

$$
\begin{aligned}
& E_{1}: x \rightarrow x_{1}, \\
& E^{1}: x \rightarrow x^{1} .
\end{aligned}
$$

Theorem 2.6. $E_{1}, E^{1}$ are projections, that is, $E_{1}, E^{1}$ are linear, continuous transformations, and $E_{1} E^{1}$ and $E^{1} E_{1}$ are both the zero transformation. Also $E_{1}^{2}=E_{1}$ and $\left(E^{1}\right)^{2}=E^{1}$.

Since $\mathfrak{X}_{1}$ is finite-dimensional (Theorem 2.2), there exists a basis $\left[x_{1}, \cdots, x_{n}\right]$ of $\mathfrak{X}_{1}$ where $\left\|x_{i}\right\|=1$ for $i=1,2, \cdots, n$ and $n$ is the dimension of $\mathfrak{X}_{1}$. Then we have:

THEOREM 2.7. There exists a set of linear functionals $f_{1}, f_{2}, \cdots, f_{n}$, elements of $\mathfrak{X}^{*}$, such that

$$
E_{1}(x)=\sum_{i=1}^{n}\left[f_{i}(x)\right] x_{i}
$$

for every $x$ an element of $\mathfrak{X}$.

Now let $g_{1}, g_{2}, \cdots, g_{n}$ be a basis for $\mathfrak{X}_{1}^{*}$ (Theorem 2.4 ). Then it can be proved that there exist elements $\bar{x}_{1}, \bar{x}_{2}, \cdots, \bar{x}_{n}$ of $\mathfrak{X}$ with the following property:

$$
g_{i}\left(\bar{x}_{j}\right)=\delta_{i j} \text { (Kronecker delta). }
$$

We define the transformation on $\mathfrak{X}: C_{0}(x)=C(x)+\sum_{i=1}^{n}\left[f_{i}(x)\right] \bar{x}_{i}$.

THEOREM 2.8. There exists $\left(I-C_{0}\right)^{-1}$ which we denote by $R$, and $R$ is linear, continuous. $\mathfrak{X}_{m}, \mathfrak{X}^{(m)}$ are invariant subspaces under the transformation $R$, and $R$ is of the form $I+Q$ where $I$ is the identity transformation and $Q$ is linear and complete continuous.

THEOREM 2.9. If $x$ is an arbitrary element of $\mathfrak{X}$, then

$$
R(I-C) x=x-E_{1} x .
$$

We shall also need an implicit function theorem for Banach spaces.

Theorem 2.10. Let $\mathfrak{X}, \mathfrak{Y}, \mathbb{Z}$ be Banach spaces. Let $U, V, W$ be open sets in $\mathfrak{X}, \mathfrak{Y}, 3$ respectively. Consider $U=U \times V \times W$ and a function $L$ defined on $U$ such that 


$$
L: \mathcal{U} \rightarrow \mathfrak{X}
$$

Let the following hypotheses be satisfied:

$\left(\mathrm{H}_{1}\right)$ There exists $\left(x_{0}, y_{0}, z_{0}\right) \in U \times V \times W$ such that

$$
x_{0}=L\left(x_{0}, y_{0}, z_{0}\right) \text {. }
$$

$\left(\mathrm{H}_{2}\right)$ There exists $k<1$ such that

$$
\left\|L\left(x_{1}, y, z\right)-L\left(x_{2}, y, z\right)\right\| \leqq k\left\|x_{1}-x_{2}\right\|
$$

for every $\left(x_{1}, y, z\right),\left(x_{2}, y, z\right)$ in $U$.

$\left(\mathrm{H}_{3}\right) L$ is uniformly continuous on the product space $U$.

Then the following conclusions hold:

$\left(C_{1}\right)$ For each $(y, z)$ in $V \times W$, there is at most one point $(x, y, z)$ in $V$ which is a solution of the equation

$$
x=L(x, y, z) \text {. }
$$

$\left(\mathrm{C}_{2}\right)$ There exist open sets $V_{1}\left(y_{0}\right)$ and $W_{1}\left(z_{0}\right)$, neighborhoods of $y_{0}$ and $z_{0}$ respectively, and a function

$$
F: V_{1} \times W_{1} \rightarrow \mathfrak{X}
$$

such that the point $(F(y, z), y, z)$ is an element of $U$ and is a solution of equation (A) for every $(y, z)$ in $V_{1} \times W_{1}$.

$\left(\mathrm{C}_{3}\right)$ The solution $F(y, z)$ of equation $(\mathrm{A})$ is uniformly continuous on its domain of definition.

This theorem is a restatement of Theorem 2, pp. 134-135 of [5].

3. The problem in Banach space. We consider the equation in Banach space $\mathfrak{X}$

$$
(I-C) x+S(y)+T(x, y)=\theta
$$

where $\theta$ is the zero element of $\mathfrak{X}, I$ is the identity mapping of $\mathfrak{X}$ onto itself, and the following assumptions are made: itself.

Assumption 3.1. $C$ is a linear, completely continuous mapping of $\mathfrak{X}$ in to

Assumption 3.2. $S$ is a continuous mapping of a subset of $\mathfrak{X}$ into $\mathfrak{X}$ with the properties:

(a) $S(\theta)=\theta$.

(b) There exists a neighborhood $N_{1}(\theta)$ of $\theta$, of radius $r_{1}$, in which $S$ satisfies a Lipschitz condition, that is, there exists a constant $B>0$ such that if $y_{1}, y_{2}$ are elements of $N_{1}(\theta)$, then

$$
\left\|S y_{1}-S y_{2}\right\| \leqq B\left\|y_{1}-y_{2}\right\| .
$$

Assumption 3.3. $T$ is a mapping of a subset of $\mathfrak{X} \times \mathfrak{X}$ into $\mathfrak{X}$ with the following properties: 
(a) $T(\theta, \theta)=\theta$.

(b) There exists a neighborhood $N_{2}(\theta)$ of $\theta$, of radius $r_{2}$, and there exists a constant $M>0$ such that if $x_{1}, x_{2}, y_{1}, y_{2}$ are elements of $N_{2}$, then $\left\|T\left(x_{1}, y_{1}\right)-T\left(x_{2}, y_{2}\right)\right\|$

$$
\leqq M\left\{\left\|x_{1}\right\|+\left\|x_{2}\right\|+\left\|y_{1}\right\|+\left\|y_{2}\right\|\right\}\left\{\left\|x_{1}-x_{2}\right\|+\left\|y_{1}-y_{2}\right\|\right\} .
$$

(c) The following $k$ th order condition is imposed upon $T$ : there exists a maximal integer $k \geqq 2$ such that if $\alpha$ is a scalar, then

$$
T[\alpha x, \theta]=\alpha^{k} T^{1}[\alpha, x]
$$

where $T^{1}$ is a continuous mapping of an open subset of the product space $Z \times \mathfrak{X}$ into $\mathfrak{X}$. ( $Z$ will denote always the space of scalars, that is, the space of complex numbers or the space of real numbers.) This subset of the product space contains points of the form $(0, x)$ where 0 is the zero of $Z$ and $x \in \mathfrak{X}$.

(It is tedious but straightforward to verify that the integral equation studied by Schmidt [17] is a special case of equation (3.1).)

The object of the discussion is to obtain information concerning the local solutions $x$ of equation (3.1) when the transformation $I-C$ is singular( $\left.{ }^{5}\right)$ and $y$ is given. This is the same as determining the local solutions $x_{1}, x^{1}$ (using the notation of Chapter 2) of the equation

$$
(I-C)\left(x_{1}+x^{1}\right)+S(y)+T\left(x_{1}+x^{1}, y\right)=\theta
$$

when $y$ is given. Since $x_{1}$ is an element of the null space of $(I-C)$, equation (3.1') may be written

$$
(I-C) x^{1}+S(y)+T\left(x_{1}+x^{1}, y\right)=\theta .
$$

Applying the transformation $R$, defined in Theorem 2.8 , to equation $\left(3.1^{\prime \prime}\right)$, we obtain

$$
R(I-C) x^{1}+R S(y)+R T\left(x_{1}+x^{1}, y\right)=\theta .
$$

Now by Theorems 2.9 and $2.6, R(I-C) x^{1}=x^{1}-E_{1} x^{1}=x^{1}$. Hence equation (3.2) becomes

$$
x^{1}=-R S(y)-R T\left(x_{1}+x^{1}, y\right) .
$$

Applying $E^{1}$, the transformation defined in Theorem 2.6, to equation (3.3) we obtain, since $E^{1} x^{1}=x^{1}$,

$$
x^{1}=-E^{1} R S(y)-E^{1} R T\left(x_{1}+x^{1}, y\right) .
$$

Applying the transformation $E_{1}$, defined in Theorem 2.6, to equation (3.3), we obtain

(5) If $(I-C)$ is nonsingular, then by multiplying through by $(I-C)^{-1}$, we put equation (3.1) in a form in which it is easy to apply Theorem 2.10 to obtain a unique local solution $x$ for given $y$ sufficiently close to $\theta$. 


$$
\theta=E_{1} R S(y)+E_{1} R T\left[x_{1}+x^{1}, y\right] .
$$

(The symbol $\theta$ will denote the zeros of $\mathfrak{X}, \mathfrak{X}_{1}$, and $\mathfrak{X}^{1}$.)

It is clear from its derivation that the system of equations (3.4) and (3.5) is equivalent to the equation $\left(3.1^{\prime \prime}\right)$ in terms of solutions $x_{1}, x^{1}$.

Now we wish to solve equation (3.4) for $x^{1}$ in terms of $y$ and $x_{1}$.

Lemma 3.1. There exist neighborhoods $U, V, W$ of $x^{1}=\theta, x_{1}=\theta$, and $y=\theta$ in $\mathfrak{X}^{1}, \mathfrak{X}_{1}$, and $\mathfrak{X}$ respectively such that the function

$$
-E^{1} R S(y)-E^{1} R T\left[x_{1}+x^{1}, y\right]
$$

may be regarded as a mapping from $U \times V \times W$ into $\mathfrak{X}^{1}$ and as such satisfies the hypotheses of Theorem 2.10 .

Proof. First, from Theorem 2.2 and 2.5, it follows that $\mathfrak{X}_{1}$ and $\mathfrak{X}^{1}$ are Banach spaces. Hypothesis $\left(\mathrm{H}_{1}\right)$ of Theorem 2.10 is satisfied because, from the definition of the transformations $C, S, T$, it follows that $x^{1}=x_{1}=y=\theta$ is the required initial solution. That hypotheses $\left(\mathrm{H}_{2}\right)$ and $\left(\mathrm{H}_{3}\right)$ are satisfied in sufficiently small neighborhoods of $x^{1}=\theta, x_{1}=\theta$, and $y=\theta$ follows from Assumptions 3.2(a), (b) and 3.3(a), (b).

It should be remarked at this point that the remainder of the discussion will concern only the domain $U \times V \times W$ or subsets thereof which will be specified later. Thus we deal only with local properties of equation (3.1).

Lemma 3.1 permits us to apply Theorem 2.10 to equation (3.4). Doing so, we obtain the unique solution

$$
x^{1}=F\left(y, x_{1}\right) .
$$

Substituting from equation (3.6) into equation (3.5), we obtain

$$
\theta=E_{1} R S(y)+E_{1} R T\left[x_{1}+F\left(y, x_{1}\right), y\right] .
$$

The system of equations (3.4) and (3.5) is equivalent to the system of equations (3.6) and (3.7). Hence we obtain finally:

LeMMA 3.2. For fixed $y$, equation (3.1") is equivalent to the pair of equations (3.6) and (3.7) in terms of solutions $x_{1}, x^{1}$.

Hence the study of the solutions $x$ of equation (3.1) is reduced to a study of the solutions $x_{1}, x^{1}$ of the pair of equations (3.6) and (3.7). Since equation (3.6) gives $x^{1}$ explicitly in terms of $x_{1}$ and $y$, we are actually concerned only with the solution $x_{1}$ of equation (3.7). Equation (3.7) is an equation in Euclidean $n$-space where $n$ is the dimension of the null space of $(I-C)$. Hence in order to obtain information concerning the solutions $x_{1}$ of equation (3.7), we investigate, for fixed $y$, the topological degree at $\theta$ of the mapping $E_{1} R S(y)$ $+E_{1} R T\left[x_{1}+F\left(y, x_{1}\right), y\right]$, which is the right side of equation (3.7) and is hence a mapping of a neighborhood of $\theta$ in $\mathfrak{X}_{1}$ into $\mathfrak{X}_{1}$. 
Definition 3.1. The multiplicity of solutions $x$ of equation (3.1) for fixed $y_{0}$ is the topological degree at $\theta$ of the mapping

$$
E_{1} R S\left(y_{0}\right)+E_{1} R T\left[x_{1}+F\left(y_{0}, x_{1}\right), y_{0}\right]
$$

relative to the open set $V_{0}$ in $\mathfrak{X}_{1}$ where $V_{0}$ is such that $V_{0} \subseteq V$.

(When we speak of the topological degree of a mapping $f$ relative to a set $M$, we shall mean the topological degree of the mapping $f / M$, that is, $f$ regarded as a mapping whose domain is $M$.)

In order to define the multiplicity, only Assumptions 3.3(a) and (b) on the transformation $T$ are used, that is, the Assumption 3.3(c) has not as yet been used.

The multiplicity (Vielfachheit) considered by R. Iglisch [7] is a special case of the multiplicity given in the definition above. Iglisch's definition is based on a consideration of an integral equation of the type dealt with by Schmidt.

To determine the topological degree, we obtain first some information concerning the form of $F\left(y, x_{1}\right)$.

Lemma 3.3. There exists a neighborhood $N(\alpha)$ of 0 in the space of scalars and a neighborhood $N\left(V_{1}\right)$ of $\theta$ in $\mathfrak{X}_{1}$ such that if $x_{1}$ is of the $\alpha v_{1}$ where $\alpha, v_{1}$ are in $N(\alpha), N\left(v_{1}\right)$ respectively, then

$$
F\left(\theta, \alpha v_{1}\right)=\alpha^{k} G\left(\alpha, v_{1}\right)
$$

where $G\left(\alpha, v_{1}\right)$ is a continuous mapping from $N(\alpha) \times N\left(v_{1}\right)$ into $\mathfrak{X}^{1}$ and $k$ is the integer described in (c) of Assumption 3.3.

Proof $\left(^{\theta}\right) . F\left(\theta, x_{1}\right)$ is the solution of equation (3.4) when $y=\theta$, that is, the equation

$$
x^{1}=-E^{1} R T\left(x_{1}+x^{1}, \theta\right) .
$$

We let $x=\alpha v_{1}$ and, for convenience, we denote the transformations $-E^{1} R T\left(x_{1}+x^{1}, \theta\right)$ and $-E^{1} R T^{\prime}\left(\alpha, x_{1}+x^{1}\right)$ by $J\left(x_{1}+x^{1}\right)$ and $J^{\prime}\left(\alpha, x_{1}+x^{1}\right)$ respectively. Then $\left(3.4^{\prime}\right)$ becomes

$$
x^{1}=J\left(\alpha v_{1}+x^{1}\right)
$$

Letting $z=x^{1} / \alpha$, we have

$$
x^{1}=J\left[\alpha\left(v_{1}+z\right)\right]=\alpha^{k} J^{\prime}\left(\alpha, v_{1}+z\right)
$$

or

$$
z=\alpha^{k-1} J^{\prime}\left(\alpha, v_{1}+z\right) .
$$

Now we apply Theorem 2.10 to solve (1) for $z$ in terms of $v_{1}$ and $\alpha$. The initial

(6) The writer is indebted to the referee for this proof which is much shorter and more direct than the original proof given. 
solution of (1) is $z=\theta, v_{1}=\theta$, and $\alpha=0$. That hypotheses $\left(\mathrm{H}_{2}\right)$ and $\left(\mathrm{H}_{3}\right)$ of Theorem 2.10 are satisfied follows from the fact that $E^{1}$ and $R$ are bounded linear transformations and from Assumption 3.3(b). Hence there exist neighborhoods $N(\alpha)$ and $N\left(v_{1}\right)$ of $\alpha=0$ and $v_{1}=\theta$ respectively such that if $\alpha \in N(\alpha)$, $v_{1} \in N\left(v_{1}\right)$, then

$$
z=A\left(\alpha, v_{1}\right)
$$

Substituting from (2) into (1), we have

$$
x^{1 / \alpha}=z=\alpha^{k-1} J^{\prime}\left[\alpha, v_{1}+A\left(\alpha, v_{1}\right)\right]
$$

or

$$
x^{1}=\alpha^{k} J^{\prime}\left[\alpha, v_{1}+A\left(\alpha, v_{1}\right)\right] .
$$

This completes the proof of Lemma 3.3.

In the discussion which follows, we shall consider not $V_{0}$, the open set of Definition 3.1, but a subset $M(\alpha) \cdot M\left(v_{1}\right)=V_{1} \subseteq V_{0}$ which has the property that $\bar{V}_{1} \subset N(a) \cdot N\left(v_{1}\right)$ where $N(a), N\left(v_{1}\right)$ are the neighborhoods described in Lemma 3.3.

Now we study two cases:

Case I. The dimension of $\mathfrak{X}_{1}$, the null space of $(I-C)$, is 1 . (Then if $\mathfrak{X}$ is over the complex numbers, $\mathfrak{X}_{1}$ is the complex plane. If $\mathfrak{X}$ is over the real numbers, $\mathfrak{X}_{1}$ is the real line.) For this case, we make

Assumption 3.4. The

$$
\lim _{\alpha \rightarrow 0} \frac{E_{1} R T\left(\alpha v_{1}, \theta\right)}{\alpha^{k}}
$$

which is equal to $E_{1} R T\left[0, v_{1}\right]$ by (c) of Assumption 3.3, is not equal to $\theta$ if $v_{1}$ is a basis for $\mathfrak{X}_{1}$ with the property that $v_{1}$ is in $V_{1}$, the neighborhood defined above. (It can be proved that this assumption becomes, for the problem considered by Schmidt, exactly Schmidt's assumption: $L_{k} \neq 0$. See $[17, \S 9]$.)

If $\mathfrak{X}$ is over the complex numbers, we have:

Lemma 3.4. The topological degree of the mapping $\alpha^{k} E_{1} R T^{1}\left[0, v_{1}\right]$ (where $\alpha$ is a complex variable) relative to an arbitrary circle in $\mathfrak{X}_{1}$ with center $\theta$ is $k$ at the point $\theta$.

If $\mathfrak{X}$ is over the real numbers, we have

Lemma 3.5. The topological degree of the mapping $\alpha^{k} E_{1} R T^{1}\left[0, v_{1}\right]$ (where $\alpha$ is a real variable) relative to an arbitrary interval in $\mathfrak{X}_{1}$ with $\theta$ as its midpoint is 0 at $\theta$ if $k$ is even and is +1 or -1 at $\theta$ if $k$ is odd.

Both of these lemmas are known.

Suppose now that $\mathfrak{X}$ is over the complex numbers. 
TheOREM 3.1. There exists a number $d_{0}>0$ and a number $r_{d}>0$ which depends on $d \leqq d_{0}$ such that if $\sigma$ is a solid circle in $\mathfrak{X}_{1}$ of radius $d \leqq d_{0}$ and center $\theta$ and if $y$ is such that $\|y\| \leqq r_{d}$, then the topological degree at $\theta$ relative to $\sigma$ of the mapping $E_{1} R S(y)+E_{1} R T\left[x_{1}+F\left(y, x_{1}\right), y\right]$ which takes a subset of $\mathfrak{X}_{1}$ into $\mathfrak{X}_{1}$ is $k$ where $k$ is the integer described in (c) of Assumption 3.3.

Proof. In this proof, we denote for convenience the transformations $E_{1} R S$, $E_{1} R T$, and $E_{1} R T^{\prime}$ by $P, J_{1}$, and $J_{1}^{\prime}$ respectively. We prove first by applying Rouché's Theorem for topological degree (see [1, p. 459]) that at the point $\theta$ and relative to a sufficiently small sphere in $\mathfrak{X}_{1}$ with center $\theta$, the mapping

$$
J_{1}\left[x_{1}+F\left(\theta, x_{1}\right), \theta\right]
$$

has topological degree $k$. We write the elements $x$ in the form $\alpha v_{1}$ where $v_{1}$ is the basis for $\mathfrak{X}_{1}$ mentioned in Assumption 3.4 and $\alpha$ is a scalar. Then by Lemma 3.3, the mapping (1) takes the form

$$
J_{1}\left[\alpha v_{1}+\alpha^{k} G\left(\alpha, v_{1}\right), \theta\right] \text {. }
$$

For convenience, we write the mapping in the form $J_{1}\left[\alpha v_{1}+\alpha^{k} G\left(\alpha, v_{1}\right)\right]$.

Now by Assumption 3.3(c), we have

$$
J_{1}\left[\alpha v_{1}+\alpha^{k} G\left(\alpha, v_{1}\right)\right]=\alpha^{k} J_{1}^{\prime}\left[\alpha, v_{1}+\alpha^{k-1} G\left(\alpha, v_{1}\right)\right] .
$$

Also by Assumption 3.3(c), $T^{\prime}$ is a continuous function. Hence if $\epsilon>0$, then there exists $\delta_{\epsilon}$ such that if $|\alpha|<\delta_{\epsilon}$ and if $\left\|x-v_{1}\right\|<\delta_{\epsilon}$, then

$$
\left\|J_{1}^{\prime}[\alpha, x]-J_{1}^{\prime}\left[0, v_{1}\right]\right\|<\epsilon .
$$

We take $\epsilon=\| J_{1}^{\prime}\left[0, v_{1}\right] \neq 0$ by Assumption 3.4. $\bar{V}_{1}$ is a closed, bounded subset of the space $Z \times \mathfrak{X}_{1}$; hence $\bar{V}_{1}$ is compact. Since $G\left(a, v_{1}\right)$ is a continuous function defined on $\bar{V}$, then $\left\|G\left(a, v_{1}\right)\right\|$ has a maximum $C \geqq 0$ on $V$. Now we require that $\alpha$ shall be contained in a spherical neighborhood $M_{1}(\alpha)$ of $\alpha=0$ with the following properties:

(1) The radius of $M_{1}(\alpha)$ is less than $\delta_{\epsilon}$, where $\epsilon=\left\|J_{1}^{\prime}\left[0, v_{1}\right]\right\|$.

(2) $M_{1}(\alpha) \subset M(\alpha)$.

(3) If $\alpha \in M_{1}(\alpha)$, then $|\alpha|^{k-1} C<\delta_{\epsilon}$ where $\epsilon=\left\|J_{1}^{\prime}\left[0, v_{1}\right]\right\|$. Then

$$
\begin{aligned}
\left\|\alpha^{k} J_{1}^{\prime}\left[\alpha, v_{1}+\alpha^{k-1} G\left(\alpha, v_{1}\right)\right]-\alpha^{k} J_{1}^{\prime}\left[0, v_{1}\right]\right\| \\
<|\alpha|^{k} \epsilon=|\alpha|{ }^{k}\left\|J_{1}^{\prime}\left[0, v_{1}\right]\right\|=\left\|\alpha^{k} J_{1}^{\prime}\left[0, v_{1}\right]\right\|
\end{aligned}
$$

for all $\alpha \in M_{1}(\alpha)$ except $\alpha=0$. In particular, inequality (2) holds for $v_{1}$ fixed to the value given in Assumption 3.4 and any circle $\sigma_{1}=\left[d /|\alpha|=d_{1}\right]$ that is contained in $M_{1}(\alpha)$. Hence by Rouché's Theorem, the topological degrees of $\alpha^{k} J_{1}^{\prime}\left[0, v_{1}\right]$ and $J_{1}\left[x_{1}+F\left(\theta, x_{1}\right), \theta\right]$ are the same at $\theta$ and relative to a solid circle $\sigma_{2}=\left[\alpha v_{1} /|\alpha| \leqq d_{1}\right]$. The number $d_{0}$ in the statement of the theorem is $\left\|d v_{1}\right\|$ where $\alpha v_{1}$ is an element in the boundary of $\sigma_{2}$. But the mapping $\alpha^{k} J_{1}^{\prime}[0$, $v_{1}$ ] has degree $k$ by Lemma 3.4 . 
Now by applying Rouché's Theorem again, we shall show that at $\theta$ and relative to a sufficiently small circle in $\mathfrak{X}_{1}$ with center $\theta$, and for sufficiently small fixed $y$, there exists a topological degree for the mapping: $P(y)$ $+J_{1}\left[x_{1}+F\left(y, x_{1}\right), y\right]$ acting on $\mathfrak{X}_{1}$, and that this topological degree is the same as the topological degree of the mapping: $J_{1}\left[x_{1}+F\left(\theta, x_{1}\right), \theta\right]$. This demonstration will complete the proof of the theorem.

As before, we write $x_{1}=\alpha v_{1}$ and keep $v_{1}$ fixed to the value given in Assumption 3.4. Now since $J_{1}\left[x_{1}+F\left(\theta, x_{1}\right), \theta\right]$ has a degree at $\theta$ and relative to any sufficiently small solid circle in $\mathfrak{X}_{1}$ with center $\theta$, then for all $x_{1}$ on the boundary of such a fixed circle $\sigma_{3} \subset M_{1}(\alpha) \cdot M\left(v_{1}\right)$,

$$
\left\|J_{1}\left[x_{1}+F\left(\theta, x_{1}\right), \theta\right]\right\|>B_{1}>0
$$

where $B_{1}$ is a constant. Also we note that the function $P(y)+J_{1}$ - $\left[x_{1}+F\left(y, x_{1}\right), y\right]$ is uniformly continuous, simultaneously in the two variables $x$ and $y$. This is because, first, $E_{1}, R, S$ are uniformly continuous transformations. Secondly, the function $F\left(y, x_{1}\right)$ is uniformly continuous simultaneously in the two variables $x_{1}$ and $y$ by conclusion $\left(\mathrm{C}_{3}\right)$ of Theorem 2.10. Finally, the transformation $T$ has the proper continuity conditions in the bounded region which we consider, by Assumption 3.3(b). Hence if $\epsilon>0$, then there exists $\delta_{\epsilon}$ such that if $\|y\|<\delta_{\epsilon}$, then

$$
\left\|P(y)+J_{1}\left[x_{1}+F\left(y, x_{1}\right), y\right]-J_{1}\left[x_{1}+F\left(\theta, x_{1}\right), \theta\right]\right\|<\epsilon
$$

for all $x_{1} \in M_{1}(a) \cdot M\left(v_{1}\right)$. Now take $\epsilon=B_{1}$. Then for $\alpha v_{1} \in \sigma_{1}$ and $y$ such that $\|y\|<\delta_{B_{1}}$,

$$
\begin{aligned}
\left\|P(y)+J_{1}\left[x_{1}+F\left(y, x_{1}\right), y\right]-J_{1}\left[x_{1}+F\left(\theta, x_{1}\right), \theta\right]\right\| & \\
<\epsilon=B_{1} & <\left\|J_{1}\left[x_{1}+E\left(\theta, x_{1}\right), \theta\right]\right\| .
\end{aligned}
$$

Applying Rouché's Theorem, we obtain the desired result where $r_{d}=\delta_{B_{1}}$. This completes the proof of the theorem.

Now suppose the Banach space $\mathfrak{X}$ is over the real numbers. Then $\mathfrak{X}_{1}$ is the real line, and we obtain:

THEOREM 3.2. There exists a number $d_{0}>0$ and a number $r_{d}>0$ which depends on $d \leqq d_{0}$ such that if $\sigma$ is an interval in $\mathfrak{X}_{1}$ of length $2 d \leqq 2 d_{0}$ and midpoint $\theta$ and $y$ is such that $\|y\|<r_{d}$, then the topological degree at $\theta$ relative to $\sigma$ of the mapping $E_{1} R S(y)+E_{1} R T\left[x_{1}+F\left(y, x_{1}\right), y\right]$ which takes $\mathfrak{X}_{1}$ into $\mathfrak{X}_{1}$ is +1 or -1 if $k$ is odd and is 0 if $k$ is even, where $k$ is the integer described in (c) of Assumption 3.3.

Proof. The proof of this theorem is carried out in the same manner as the proof of Theorem 3.1 except that in this case, Lemma 3.5, instead of Lemma 3.4, is applied.

Thus the multiplicity of Definition 3.1 is determined if $y$ and $x_{1}$ are suffi- 
ciently restricted. We may summarize the results of the preceding discussion as follows:

THeOREM 3.3. There exist numbers $r_{1}>0$ and $r_{2}>0$ such that if $\left\|y_{0}\right\|<r_{1}$, then in the complex case, there exist $k$ solutions $x$ (in the sense of the multiplicity of Definition 3.1) for this $y_{0}$ and each solution $x$ is such that $\|x\|<r_{2}$. In the real case, there exist $1,-1$, or 0 solutions $x$ such that $\|x\|<r_{2}$.

Definition 3.2. If $k$ is the multiplicity of solutions, we call the point $x=\theta, y=\theta$ a branch point of order $k$ of equation (3.1).

Case II. The dimension of $\mathfrak{X}_{1}$, the null space of $(I-C)$, is greater than 1 .

For this case, we make the following additional assumptions concerning the transformation $T$ :

Assumption 3.5. The transformation $T$ is split into a term of order $k$ $(k \geqq 2)$ in $x$ and a term of higher order, that is,

$$
T(x, \theta)=T_{k}(x)+T_{k+1}(x)
$$

and

(a) $T_{k}$ is homogeneous of degree $k$ in $x$, that is, if $m$ is an arbitrary integer,

$$
T_{k}\left(\sum_{i=1}^{m} \alpha_{i} \omega_{i}\right)=\sum \alpha_{1}^{\beta_{1}} \cdots \alpha_{m}^{\beta_{m}} T_{\beta_{1} \cdots \beta_{m}}\left(\omega_{1}, \cdots, \omega_{m}\right)
$$

where the summation is taken over all $\beta_{i}$ such that $\beta_{i} \geqq 0$ and $\sum_{i=1}^{m} \beta_{i}=k$, and $T_{\beta_{1} \cdots \beta_{m}}\left(\omega_{1}, \cdots, \omega_{m}\right)$ is a continuous mapping from $\mathfrak{X} \times \cdots \times \mathfrak{X}$ into $\mathfrak{X}$ which is independent of $\omega_{i}$ if $\beta_{i}=0$. (From this assumption, it follows that if $\beta_{i}=k, T_{\beta_{1}} \ldots \beta_{m}=T_{k}\left(\omega_{i}\right)$.)

(b) There exists $\lim _{\omega \rightarrow \theta} T_{k+1}(\omega, \theta) /\|\omega\|^{k}$, and this limit is zero.

Assumption 3.6. The element $\theta$ is an isolated $\theta$-point of the transformation $E_{1} R T_{k}[x]$. We may express this assumption also in the following way: the only $\theta$-point of $\mathfrak{X}_{1}$ under the mapping $E_{1} R T_{k}[x]$ is $\theta$. This seemingly stronger condition is actually equivalent to the first mentioned condition because of (a) in Assumption 3.5, that is, $T_{k}[\alpha x, \theta]=\alpha^{k} T_{k}[x, \theta]$.

LemMA 3.6. The mapping $E_{1} R T_{k}[x]$ acting on $\mathfrak{X}_{1}$ has a topological degree defined at the point $\theta$ and relative to an arbitrary sphere in $\mathfrak{X}_{1}$ having $\theta$ as its center.

Proof. This is an immediate consequence of the definition of topological degree and Assumption 3.6.

THEOREM 3.4. There exists a number $d_{0}>0$ and a number $r\left(d_{2}\right)>0$ which depends on $d_{2} \leqq d_{0}$ such that if $\sigma$ is a solid sphere in $\mathfrak{X}_{1}$ of radius $d_{2} \leqq d_{0}$ and center $\theta$ and $y$ is such that $\|y\|<r\left(d_{2}\right)$, then the topological degree at $\theta$ relative to $\sigma$ of the mapping 


$$
E_{1} R S(y)+E_{1} R T\left[x_{1}+F\left(y, x_{1}\right), y\right]
$$

is equal to the topological degree at $\theta$ of the mapping $E_{1} R T_{k}\left[x_{1}\right]$ acting on $\mathfrak{X}_{1}$, relative to $\sigma$.

Proof. In this proof, we use the notations $P$ and $J_{1}$ introduced in the proof of Theorem 3.1 and we use the notations $J_{1 k}, J_{1, k+1}$, and $J_{1 p, q}$ in place of $E_{1} R T_{k}, E_{1} R T_{k+1}$, and $E_{1} R T_{p, q}$ respectively. We prove first that for a sufficiently small sphere, the mapping

$$
J_{1}\left[x_{1}+F\left(\theta, x_{1}\right), \theta\right]
$$

has the same topological degree as $J_{1 k}\left[x_{1}\right]$. For convenience, we write the mapping (1) in the form $J_{1}\left[x_{1}+F\left(\theta, x_{1}\right)\right]$. Also we write $x_{1}$ in the form $\alpha v_{1}$ where $\alpha, v_{1}$ are in $M(\alpha), M\left(v_{1}\right)$, the neighborhoods defined immediately after the proof of Lemma 3.3. By Lemma 3.3,

$$
\left\|J_{1 k}\left[\alpha v_{1}\right]-J_{1}\left[\alpha v_{1}+F\left(\theta, \alpha v_{1}\right)\right]\right\|=\left\|J_{1 k}\left[\alpha v_{1}\right]-J_{1}\left[\alpha v_{1}+\alpha^{k} G\left(\alpha, v_{1}\right)\right]\right\| .
$$

But

$$
\begin{aligned}
\| J_{1 k}\left[\alpha v_{1}\right] & -J_{1}\left[\alpha v_{1}+\alpha^{k} G\left(\alpha, v_{1}\right)\right] \| \\
= & |\alpha|^{k}\left\|J_{1 k}\left[v_{1}\right]-J_{1 k}\left[v_{1}+\alpha^{k-1} G\left(\alpha, v_{1}\right)\right]-\frac{1}{\alpha^{k}} J_{1, k+1}\left[\alpha v_{1}+\alpha^{k} G\left(\alpha, v_{1}\right)\right]\right\| \\
= & |\alpha|^{k}\left\{\| J_{1 k}\left[v_{1}\right]-J_{1 k}\left[v_{1}\right]-\sum_{p+q=k ; p, q=1}^{k-1}\left(\alpha^{k-1}\right)^{q} J_{1 p, q}\left[v_{1}, G\left(\alpha v_{1}\right)\right]\right. \\
& \left.\quad-\left(\alpha^{k-1}\right)^{k} J_{1 k}\left[G\left(\alpha, v_{1}\right)\right]-\frac{1}{\alpha^{k}} J_{1, k+1}\left[\alpha v_{1}+\alpha^{k} G\left(\alpha, v_{1}\right)\right] \|\right\}
\end{aligned}
$$

by Assumption 3.5. Therefore

$$
\begin{aligned}
\| J_{1 k}\left[\alpha v_{1}\right]- & J_{1}\left[\alpha v_{1}+\alpha^{k} G\left(\alpha, v_{1}\right)\right] \| \leqq|\alpha|^{k}\left\{\sum_{p, q}\left|\alpha^{k-1}\right| q|| J_{1 p, q}\left[v_{1}, G\left(\alpha, v_{1}\right)\right] \|\right. \\
& \left.+\left|\alpha^{k-1}\right| k|| J_{1 k}\left[G\left(\alpha, v_{1}\right)\right]\left\|+\frac{1}{|\alpha|^{k}}\right\| J_{1, k+1}\left[\alpha v_{1}+G\left(\alpha, v_{1}\right)\right] \|\right\} .
\end{aligned}
$$

Since

$\left\|J_{1 k}\left[\alpha v_{1}\right]-J_{1}\left[\alpha v_{1}+\alpha^{k} G\left(\alpha, v_{1}\right)\right]\right\|=|\alpha| k|| J_{1 k}\left[v_{1}\right]-J_{1}^{\prime}\left[\alpha, v_{1}+\alpha^{k-1} G\left(\alpha, v_{1}\right)\right] \|$, inequality (2) becomes, when divided by $|\alpha|^{k}$ :

$$
\begin{aligned}
\| J_{1 k}\left[v_{1}\right]- & J_{1}^{\prime}\left[\alpha, v_{1}+\alpha^{k-1} G\left(\alpha, v_{1}\right)\right]\left\|\leqq \sum_{p, q}\left|\alpha^{k-1}\right| q\right\| J_{1 p, q}\left[v_{1}, G\left(\alpha, v_{1}\right)\right] \| \\
& +\left|\alpha^{k-1}\right| k|| J_{1 k}\left[G\left(\alpha, v_{1}\right)\right]\left\|+\frac{1}{|\alpha|^{k}}\right\| J_{1, k+1}\left[\alpha v_{1}+\alpha^{k} G\left(\alpha, v_{1}\right)\right] \| .
\end{aligned}
$$


$G\left(\alpha, v_{1}\right)$ is, by Lemma 3.3 , a continuous function of $\alpha$ and $v_{1}$, and $J_{1 k}$ is a continuous transformation. Hence if

$$
\left\|J_{1 k}[G(0, \theta)]\right\| \leqq B_{2}>0,
$$

then there exist spherical neighborhoods $M_{2}(\alpha) \subset M(\alpha)$ of $\alpha=0$ and $M_{2}\left(v_{1}\right)$ of $v_{1}=\theta$ such that if $\alpha \in M_{2}(\alpha), v_{1} \in M_{2}\left(v_{1}\right)$, then $\left\|J_{1 k}\left[G\left(\alpha, v_{1}\right)\right]\right\|<2 B_{2}$.

Also

$$
\begin{aligned}
\frac{1}{|\alpha|^{k}}\left\|J_{1, k+1}\left[\alpha v_{1}+\alpha^{k} G\left(\alpha, v_{1}\right)\right]\right\| & \\
=\left\|v_{1}+\alpha^{k-1} G\left(\alpha, v_{1}\right)\right\| k & \left\{\frac{\left\|J_{1, k+1}\left[\alpha v_{1}+\alpha^{k} G\left(\alpha, v_{1}\right)\right]\right\|}{|\alpha|^{k}\left\|v_{1}+\alpha^{k-1} G\left(\alpha, v_{1}\right)\right\| k}\right\} \\
& =\left\|v_{1}+\alpha^{k-1} G\left(\alpha, v_{1}\right)\right\| k\left\{\frac{\left\|J_{1, k+1}\left[\alpha v_{1}+\alpha^{k} G\left(\alpha, v_{1}\right)\right]\right\|}{\left\|\alpha v_{1}+\alpha^{k} G\left(\alpha, v_{1}\right)\right\| k}\right\} .
\end{aligned}
$$

There exist spherical neighborhoods $M_{3}(\alpha) \subset M_{2}(\alpha)$ and $M_{3}\left(v_{1}\right) \subset M_{2}\left(v_{1}\right)$ of $\alpha=0$ and $v_{1}=\theta$, respectively, such that if $v_{1} \in M_{3}\left(v_{1}\right)$ and $\alpha \in M_{3}(\alpha)$, then

$$
\left\|v_{1}+\alpha^{k-1} G\left(\alpha, v_{1}\right)\right\|<2 r
$$

where $r$ is the radius of $M_{3}\left(v_{1}\right)$.

Finally we deal with the term $\sum_{p, q}\left|\alpha^{k-1}\right| q\left\|J_{1 p, q}\left[v_{1}, G\left(\alpha, v_{1}\right)\right]\right\|$. By Assumption 3.5(b), each function $T_{p, q}$ is continuous in $v_{1}$ and $G\left(\alpha, v_{1}\right)$. Hence if $J_{1 p, q}[\theta, G(0, \theta)] \leqq M_{p, q}>0$, then there exist spherical neighborhoods $M_{4}(\alpha) \subset M_{3}(\alpha), M_{4}\left(v_{1}\right) \subset M_{3}\left(v_{1}\right)$ of $\alpha=0$ and $v_{1}=\theta$ respectively such that if $\alpha \in M_{4}(\alpha), v_{1} \in M_{4}\left(v_{1}\right)$, then

$$
\left\|J_{1 p, q}\left[v_{1}, G\left(\alpha, v_{1}\right)\right]\right\|<2 M_{p, q}
$$

for each $J_{1 p, q}$.

Summarizing these results, we have: there exist spherical neighborhoods $U(\alpha), U\left(v_{1}\right)$ of $\alpha=0, v_{1}=\theta$ respectively such that if $\alpha \in U(\alpha), V_{1} \in U\left(v_{1}\right)$, then

$$
\begin{aligned}
\left\|J_{1 k}\left[G\left(\alpha, v_{1}\right)\right]\right\| & <2 B_{2}, \\
\left\|v_{1}+\alpha^{k-1} G\left(\alpha, v_{1}\right)\right\| & <2 r, \\
\left\|J_{1 p, q}\left[v_{1}, G\left(\alpha, v_{1}\right)\right]\right\| & <2 M_{p, q},
\end{aligned}
$$

where $B_{2}, r, M_{p, q}$ are positive constants. Hence by inequality (3) and equation (4),

$$
\begin{aligned}
\left\|J_{1 k}\left[v_{1}\right]-J_{1}^{\prime}\left[\alpha, v_{1}+\alpha^{k-1} G\left(\alpha, v_{1}\right)\right]\right\| \leqq & \sum_{p, q}\left|\alpha^{k-1}\right| q M_{p, q}+\left|\alpha^{k-1}\right|{ }^{k} 2 B_{2} \\
& +(2 r)^{k} \frac{\left\|J_{1, k+1}\left[\alpha v_{1}+\alpha^{k} G\left(\alpha, v_{1}\right)\right]\right\|}{\left\|\alpha v_{1}+\alpha^{k} G\left(\alpha, v_{1}\right)\right\|^{k}}
\end{aligned}
$$


Now consider the sphere $\sigma$ in $\mathfrak{X}_{1}:\left[v_{1} /\left\|v_{1}\right\|=d\right]$, where $d<r_{3}$, the radius of $U\left(v_{1}\right)$. Since $\sigma$ is compact, then $\left\|J_{1, k}\left[v_{1}\right]\right\|$ has a minimum on this set. But this minimum $m$ is greater than zero by Assumption 3.6.

Now there exists a spherical neighborhood $U_{1}(\alpha) \subset U(\alpha)$ such that if $\alpha \in U_{1}(\alpha)$, then

$$
\sum_{p, q}\left|\alpha^{k-1}\right|{ }^{q} M_{p, q}+\left|\alpha^{k-1}\right|{ }^{k} 2||_{2}<\frac{m}{2} .
$$

Also by Assumption 3.5(b)

$$
\lim _{\omega \rightarrow \theta} \frac{T_{k+1}[\omega, \theta]}{\|\omega\|^{k}}=0 .
$$

Hence there exists $\delta>0$ such that if $\left\|\alpha v_{1}+\alpha^{k} G\left(\alpha, v_{1}\right)\right\|<\delta$, then

$$
\frac{\left\|J_{1, k+1}\left[\alpha v_{1}+\alpha^{k} G\left(\alpha, v_{1}\right)\right]\right\|}{\left\|\alpha v_{1}+\alpha^{k} G\left(\alpha, v_{1}\right)\right\|^{k}}<\frac{m}{2(2 r)^{k}} .
$$

There exists a spherical neighborhood $U_{2}(\alpha) \subset U_{1}(\alpha)$ of $\alpha=0$ such that if $\alpha \in U_{2}(\alpha)$, then $|\alpha|(2 r)<\delta$, and hence by inequality (6), $\| \alpha v_{1}+\alpha^{k} G\left(\alpha, v_{1} \|<\delta\right.$ for $\alpha \in U_{2}(\alpha)$ and $v_{1} \in U\left(v_{1}\right)$.

Hence if $\alpha \in U_{2}(\alpha)$, and $\left\|v_{1}\right\|=d$, then

$$
\begin{aligned}
& \left\|J_{1 k}\left[v_{1}\right]-J_{1}^{\prime}\left[\alpha, v_{1}+\alpha^{k-1} G\left(\alpha, v_{1}\right)\right]\right\| \\
& \quad \leqq \sum_{p, q}\left|\alpha^{k-1}\right|{ }^{q} M_{p, q}+\left|\alpha^{k-1}\right|{ }^{k} 2 B_{2}+(2 r)^{k}\left\{\frac{\left\|J_{1, k+1}\left[\alpha v_{1}+\alpha^{k} G\left(\alpha, v_{1}\right)\right]\right\|}{\left\|\alpha v_{1}+\alpha^{k} G\left(\alpha, v_{1}\right)\right\| k}\right\} \\
& \quad<\frac{m}{2}+(2 r)^{k}\left[\frac{m}{2(2 r)^{k}}\right]=m \leqq\left\|J_{1 k}\left[v_{1}\right]\right\| .
\end{aligned}
$$

Multiplying inequality (8) by $\left|\alpha^{k}\right|$, we obtain

$$
\left\|J_{1 k}\left[\alpha v_{1}\right]-J_{1}\left[\alpha v_{1}+\alpha^{k} G\left(\alpha, v_{1}\right)\right]\right\|<\left\|J_{1 k}\left[\alpha v_{1}\right]\right\|
$$

for $\alpha \in U_{2}(\alpha)$ and $\left\|v_{1}\right\|=d$. Hence by Lemma 3.5, the topological degree relative to the sphere

$$
\sigma=\left[\alpha v_{1} /\left\|v_{1}\right\|=d,|\alpha|=d_{1}\right]
$$

at the point $\theta$ of the mapping $J_{1 k}\left[d v_{1}\right]$ is the same as the topological degree of $J_{1}\left[\alpha v_{1}+\alpha^{k} G\left(\alpha, v_{1}\right)\right]$. The number $d_{0}$ in the statement of the theorem is $\left(r_{3}\right)\left(r_{4}\right)$. The topological degree of $J_{1}\left[\alpha v_{1}+\alpha^{k} G\left(\alpha, v_{1}\right)\right]$ is the same as the topological degree of $P(y)+J_{1}\left[\alpha v_{1}+F\left(y, \alpha v_{1}\right), y\right]$ by the same proof as is used for the proof of the analogous statement in Theorem 3.1. This completes the proof of the theorem.

It will be noted that the discussion up to this point is quite general, that is, the statements proved so far hold regardless of the dimension of $\mathfrak{X}_{1}$. Hence 
if Assumption 3.6 holds, Theorem 3.2 would apply if the dimension of $\mathfrak{X}_{1}$ were 1 . If the dimension of $\mathfrak{X}_{1}$ were 1 , Assumption 3.6 could be replaced by the simpler assumption: $\left\|E_{1} R T_{k}\left[v_{1}, \theta\right]\right\| \neq \theta$ where $v_{1}$ is any basis for $\mathfrak{X}_{1}$.

Now we have the general problem of determining the topological degree of $\theta$ of the mapping $E_{1} R T_{k}[x]$ of a subset of $\mathfrak{X}_{1}$ into $\mathfrak{X}_{1}$. First $\mathfrak{X}_{1}$ is an $n$-dimensional Euclidean space complex or real according as $\mathfrak{X}$ is complex or real. Hence $E_{1} R T_{k}\left[x_{1}\right]$ is a mapping of a subset of Euclidean $n$-space into itself. Let $x_{1}, x_{2}, \cdots, x_{n}$ be a basis for $\mathfrak{X}_{1}$ such that

$$
\left\|x_{i}\right\|=d_{2}, \quad i=1,2, \cdots, n,
$$

where $d_{2}$ is the number defined in Theorem 3.4. Then each element $x$ in $\mathfrak{X}_{1}$ is of the form: $x=\sum_{i=1}^{n} \alpha_{i} x_{i}$ where $\alpha_{i}$ is a complex or real number $(i=1, \cdots, n)$.

Then by Assumption 3.5, we may write

$$
\begin{aligned}
E_{1} R T_{k}(x) & =E_{1} R T_{k} \sum_{i=1}^{n} \alpha_{i} x_{i} \\
& =\sum_{i=1}^{n} \alpha_{i}^{k} E_{1} R T_{k}\left[x_{i}\right]+\sum \alpha_{1}^{\beta_{1}} \cdots \alpha_{n}^{\beta_{n}} E_{1} R T_{\beta_{1}} \cdots \beta_{n}\left[x_{1}, \cdots, x_{n}\right]
\end{aligned}
$$

where the second sum is taken over non-negative integers $\beta_{i}$ such that $\sum_{i=1}^{n} \beta_{i}=k$. The terms $E_{1} R T_{k}\left[x_{i}\right]$ and $T_{\beta_{1}} \cdots \beta_{n}\left[x_{1}, \cdots, x_{n}\right]$ are fixed elements of $\mathfrak{X}_{1}$. The $\alpha_{i}$ 's are variables. Now if $P_{j}$ is the projection of $\mathfrak{X}_{1}$ onto the subspace with basis $x_{j}(j=1, \cdots, n)$, then the mapping may be written in the form

(1-j) $\quad \sum_{i=1}^{n} \alpha_{i}^{n} P_{j} E_{1} R T_{k}\left[x_{i}\right]+\sum_{\beta_{1}} \alpha_{1}^{\beta_{1}} \cdots \alpha_{n}^{\beta_{n}} P_{j} E_{1} R T_{\beta_{1} \cdots \beta_{n}}\left[x_{1}, \cdots, x_{n}\right]=b_{j} x_{j}$

where $j=1,2, \cdots, n$. Thus the general problem is to determine the topological degree of a mapping which is given by $n$ polynomials in $n$ variables, each polynomial being homogeneous of degree $k$ and having complex or real coefficients.

Some special cases of this problem were considered in my doctoral thesis. More general results will be described in a later paper. We point out here only two simple examples for which the topological degree is easily computed.

If $\mathfrak{X}$ is over the complex numbers, then the mapping of $\mathfrak{X}_{1}$ into itself,

$$
\begin{array}{r}
a_{1} z_{1}=z_{1}^{\prime}, \\
a_{2} z_{2}=z_{2}^{\prime}, \\
\cdot \cdot \cdot \cdot, \\
a_{n} z_{n}^{k}=z_{n}^{\prime},
\end{array}
$$

has topological degree $k^{n}$ at $(0,0, \cdots, 0)$. 
If $\mathfrak{X}$ is over the real numbers, then the mapping

$$
\begin{aligned}
a_{1} x_{1} & =x_{1}^{\prime}, \\
a_{2} x_{1}^{k} & =x_{2}^{\prime}, \\
\cdot \cdot & \cdot \\
a_{n} x_{n}^{k} & =x_{n}^{\prime},
\end{aligned}
$$

has degree 0 if $k$ is even and degree 1 or -1 if $k$ is odd.

If the mapping $(1-j)$ is of the form $(2-j)$ in the complex case or of the form $(3-j)$ in the real case, we have:

THEOREM 3.5. There exist numbers $r_{1}>0$ and $r_{2}>0$ such that if $\left\|y_{0}\right\|<r_{1}$, then if $\mathfrak{X}$ is over the complex numbers, there exist $k^{n}$ solutions $x$ (in the sense of the multiplicity of Definition 3.1) of equation (3.1) for this $y_{0}$ and each solution $x$ is such that $\|x\|<r_{2}$. If $\mathfrak{X}$ is over the real numbers, there exist $1,-1$, or 0 solutions $x$ such that $\|x\|<r_{2}$.

4. Applications of the theory. We obtain now an extension of a special case of Schmidt's work by considering the following functional equation:

$$
x(s)+\int_{a}^{b} G(s, t)\{F[x(t), y(t)]\} d t=0 .
$$

We wish to study the solutions $x(s)$ of equation (4.1) if $G(s, t), F(x, y)$, and $y(t)$ are given continuous functions of their arguments. It is assumed that equation (4.1) has an initial solution, that is, that there exist functions $x_{0}(t), y_{0}(t)$ continuous in the closed interval $(a, b)$ such that

$$
x_{0}(s)+\int_{a}^{b} G(s, t)\left\{F\left[x_{0}(t), y_{0}(t)\right]\right\} d t=0 .
$$

Subtracting equation (1) from equation (4.1), we obtain

$$
x(s)-x_{0}(s)+\int_{a}^{b} G(s, t)\left\{F[x(t), y(t)]-F\left[x_{0}(t), y_{0}(t)\right]\right\} d t=0 .
$$

We obtain a result which is an extension of Schmidt's work in the following sense: Schmidt deals only with an infinite series. Hence in order to apply Schmidt's work to the problem of solving equation (4.2), it is necessary to assume that $F(x, y)$ is an analytic function of the two variables $x, y$. In order to apply the theory of $\S 3$, it is only necessary to assume that the function $F(x, y)$ has second or third derivatives in the two variables.

We introduce first the following constants. Let $\epsilon$ be a positive number, and let $A, B, C, D$ be the numbers:

$$
A=\min _{a \leqq t \leqq b}\left[x_{0}(t)-\epsilon\right], \quad B=\max _{a \leqq t \leqq b}\left[x_{0}(t)+\epsilon\right],
$$




$$
C=\min _{a \leqq t \leqq b}\left[y_{0}(t)-\epsilon\right], \quad D=\max _{a \leqq t \leqq b}\left[y_{0}(t)+\epsilon\right] .
$$

THEOREM 4.1. If the third derivatives of the function $F(x, y)$ exist and satisfy Lipschitz conditions for $A \leqq x \leqq B, C \leqq x \leqq D$, then equation (4.2) satisfies $A s$ sumptions 3.1, 3.2, 3.3, and 3.5 of \$3. Hence the discussion of Case II in $\$ 3$ applies to equation (4.2) and consequently to equation (4.1).

Proof. By hypothesis, we may write

$$
\begin{aligned}
F(x, y)-F\left(x_{0}, y_{0}\right)= & \Delta x\left[F_{x}\left(x_{0}, y_{0}\right)\right]+\Delta y\left[F_{y}\left(x_{0}, y_{0}\right)\right]+\frac{(\Delta x)^{2}}{2 !} F_{x x}\left(x_{0}, y_{0}\right) \\
& +(\Delta x)(\Delta y) F_{x y}\left(x_{0}, y_{0}\right)+\frac{(\Delta y)^{2}}{2 !} F_{y y}\left(x_{0}, y_{0}\right)+R_{3}
\end{aligned}
$$

where $R_{3}$ is the remainder in the Taylor's expansion and $\Delta x=x-x_{0}, \Delta y=y$ $-y_{0} . R_{3}$ may be written in the form

$$
\begin{aligned}
R_{3}= & \frac{1}{2 !} \int_{0}^{1}\left[(\Delta x)^{3} F_{x x x}\left(x_{0}+\tau \Delta x, y_{0}+\tau \Delta y\right)\right. \\
& +3(\Delta x)^{2}(\Delta y) F_{x x y}\left(x_{0}+\tau \Delta x, y_{0}+\tau \Delta y\right) \\
& +3(\Delta x)(\Delta y)^{2} F_{x y y}\left(x_{0}+\tau \Delta x, y_{0}+\tau \Delta y\right) \\
& \left.+(\Delta y)^{3} F_{y y y}\left(x_{0}+\tau \Delta x, y_{0}+\tau \Delta y\right)\right](1-\tau) d \tau .
\end{aligned}
$$

Hence equation (4.2) takes the form

$$
\begin{aligned}
\Delta x & +\int_{a}^{b} G(s, t)\left\{\Delta x\left[F_{x}\left(x_{0}, y_{0}\right)\right]+\Delta y\left[F_{y}\left(x_{0}, y_{0}\right)\right]+\frac{(\Delta x)^{2}}{2 !} F_{x x}\left(x_{0}, y_{0}\right)\right. \\
& \left.+(\Delta x)(\Delta y) F_{x y}\left(x_{0}, y_{0}\right)+\frac{(\Delta y)^{2}}{2 !} F_{y y}\left(x_{0}, y_{0}\right)+R_{3}\right\} d t=0 .
\end{aligned}
$$

With the equation in this form, it is a matter of straightforward but tedious verification to complete the proof of the theorem.

THEOREM 4.2. If the second derivatives of the function $F(x, y)$ exist and satisfy Lipschitz conditions for

$$
A \leqq x \leqq B, \quad C \leqq y \leqq D,
$$

then equation (4.2) satisfies Assumptions 3.1, 3.2, 3.3. If, in addition, the null space of the transformation

$$
\Delta x+\int_{a}^{b} G(s, t) F_{x}\left(x_{0}, y_{0}\right) \Delta x d t
$$

acting on $\Delta x$, has dimension one, then the discussion of Case I in $\$ 3$ applies to 
equation (4.2) and consequently to equation (4.1).

Proof. The same type of verification as for Theorem 4.1 is required here.

The theory of $\$ 3$ permits us to deal with equation (4.2) if the functions $F$ and $G$ are complex or if they are real. In the real case, equation (4.2) is a generalization of the integral equation treated by R. Iglisch. (See equation (31), p. 107 of [6].) Following Iglisch, we may apply the information concerning the solutions $x(s)$ of (4.2) obtained from our theory to the study of a differential equation of the form

$$
\Delta u=F(u) .
$$

Iglisch assumes that $F(u)$ is real and analytic in $u$. In order to apply Theorems 4.1 and 4.2 , it is necessary to assume only that $F(u)$ has second or third derivatives. Hence a generalization of Iglisch's results may be obtained. This generalization is not obtained without some sacrifice however. In the case when the dimension of the space $\mathfrak{X}_{1}$ is one, Iglisch showed that equation (4.3) has a unique solution if $k$ is odd and no solution if $k$ is even. By using the multiplicity of Definition 3.1, we obtain the result that if $k$ is odd, there is at least one solution. If $k$ is even, the multiplicity is zero, and hence we obtain no definite information about the existence of solutions. The possibility of obtaining results as sharp as those of Iglisch for this more general case is not excluded. The theory of topological degree has been used to obtain proofs of uniqueness theorems (see [14]). But the theory of $\$ 3$ does not yield such sharp results immediately.

When the dimension of $\mathfrak{X}_{1}$ is greater than one, the results obtained by application of the theory in $\$ 3$ are more general than those obtained by Iglisch [8], for Iglisch deals almost entirely with the special case $n=k=2$.

5. An extension of the Leray-Schauder definition of degree of a mapping in Banach space. In this section, we establish a connection between the multiplicity of Definition 3.1 in $\$ 3$ and the topological degree defined by Leray and Schauder [10].

We consider the equation in Banach space $\mathfrak{X}$

$$
(I-C) x+T(x)=y \text { or }(I-C+T) x=y
$$

which is a special case of equation (3.1), that is, $I$ is the identity transformation, $C$ is linear, completely continuous, the transformation $T$ satisfies the conditions of Assumption 3.3 and is independent of $y$, and $S(y)=-y$. It will be proved here that if the Leray-Schauder topological degree is defined for the transformation $I-C+T$ (that is, if the transformation $T$ is completely continuous), then the multiplicity of solutions $x$ for equation (5.1) for fixed $y_{0}$ as given by Definition 3.1 is equal to the Leray-Schauder degree of $I-C+T$ at $y_{0}$ or differs from it by a factor $(-1)$.

First we remark that the multiplicity given in Definition 3.1 has some of the properties of a topological degree. If the topological degree at $y$ of a 
mapping $H$, relative to a solid sphere $\sigma$, is different from zero, then there exists an element $x$ of $\sigma$ such that $H(x)=y$. From the corresponding statement for the topological degree of a mapping from Euclidean $n$-space into Euclidean $n$-space, it follows that this condition holds for the multiplicity we have defined. Also the topological degree is the same relative to all $y$ in a sufficiently small neighborhood of $y_{0}$. If the transformation $T$ satisfies the conditions of Assumption 3.4 or of Assumptions 3.5 and 3.6, then this property holds for the multiplicity. (See Theorems 3.1 and 3.4 of $\S 3$.)

Before demonstrating the relationship between the Leray-Schauder degree and the multiplicity of Definition 3.1, we exhibit a transformation $I-C+T$ for which the Leray-Schauder degree is not defined but for which our multiplicity is defined. Thus we show that the multiplicity of Definition 3.1 is a true extension of the Leray-Schauder definition of degree $\left({ }^{7}\right)$.

The Leray-Schauder topological degree is defined for mappings of the form $(I+D)$ where $I$ is the identity transformation and $D$ is completely continuous (not necessarily linear). Hence we must exhibit a transformation $(I-C+T)$ such that $T$ is not completely continuous but for which our definition of multiplicity holds.

We consider the Banach space $\mathfrak{V}$ of complex-valued continuous functions defined on the closed interval $(0,1)$ where, if $x(t) \in \mathfrak{Y}$, then

$$
\|x(t)\|=\max _{0 \leqq t \leqq 1}|x(t)| \text {. }
$$

The transformation $C$ is the finite-dimensional (hence completely continuous) mapping which takes $x(t)$ into the function $y(t)=[x(1)] t$. $C$ is clearly linear and continuous. Also $C$ maps $\mathfrak{V}$ into a one-dimensional linear subspace $\mathfrak{R}$ of $\mathfrak{Y}$. From the definition of $C$, it follows that the null space $\mathfrak{X}_{1}$ of $(I-C)$ is $\mathbb{R}$, hence is one-dimensional. The transformation $T$ is defined as

$$
T: x(t) \rightarrow[x(t)]^{2} .
$$

$T$ is continuous. $T(\theta)=\theta$. Also the transformation $T$ is not completely continuous. It is trivial to verify that the transformation $T$ satisfies Assumption 3.3. The transformation $T^{\prime}[a, x(t)]$ is simply $T[x(t)]$. It is also easy to verify that Assumption 3.4 is satisfied by showing that $\mathfrak{X}_{m}=\mathfrak{X}_{1}$ in this case and by using the fact that $\mathfrak{X}_{m}$ is invariant under $R$ (Theorem 2.8 ).

We establish now the relationship between the Leray-Schauder topological degree and the multiplicity of Definition 3.1. For the proof, we require two lemmas concerning the Leray-Schauder topological degree.

(7) The word "extension" is not used in the sense of "generalization" here. The multiplicity of Definition 3.1 is not a generalization of the Leray-Schauder degree. First, it has not been proved that the multiplicity is invariant under homotopy. Secondly, the multiplicity is defined only if the transformation has a differential, while the Leray-Schauder degree is defined for an arbitrary mapping of the form $I+D$ where $D$ is completely continuous. 
Lemмa 5.1. Let $\Phi=I+H$ and $\Psi=I+G$ be two mappings of subsets of Banach space $\mathfrak{X}$ into $\mathfrak{X}$ such that:

(a) I is the identity mapping on $\mathfrak{X} ; H, G$ are completely continuous mappings of subsets of $\mathfrak{X}$ into $\mathfrak{X}$.

(b) $\omega$ is a bounded open set with boundary $\omega^{\prime}$ such that $H$ is defined on $\bar{\omega}=\omega \cup \omega^{\prime}$ and $G$ is defined on the closure $A$ of a bounded open set containing $(I+H)(\bar{\omega})$.

We denote by $d$ a bounded component of $\mathfrak{X}-\Phi\left(\omega^{\prime}\right)$. If the point $p$ is not in $\Psi \Phi\left(\omega^{\prime}\right)$ and is not in $\Psi$ [Boundary $\left.\Phi(\omega)\right]$, and if $[\Psi \Phi, \omega, p]$ is defined, then only a finite number of the terms $[\Phi, \omega, d] \cdot[\Psi, d, p]$ are nonzero, and

$$
[\Psi \Phi, \omega, p]=\sum_{(d)}[\Phi, \omega, d] \cdot[\Psi, d, p]
$$

where $\sum_{(d)}$ means the summation over the bounded components $d,[\Psi \Phi, \omega, p]$ is the Leray-Schauder topological degree of the point $p$ of the mapping $\Psi \Phi$, relative to $\omega$, and $[\Phi, \omega, d],[\Psi, d, p]$ have similar meanings.

Proof. This lemma is known. See [9] for an outline of the proof.

In order to apply Lemma 5.1 here, it will be necessary to consider a domain $\omega$ such that there is just one nonzero term in $\sum_{(d)}[\Phi, \omega, d][\Psi, d, c]$.

LemMA 5.2. If $\Phi(\theta)=\theta$ and $\theta$ is an isolated $\theta$-point of $\Psi$, that is, there exists a neighborhood $U(\theta)$ of $\theta$ such that if $x$ is an element of $U$ and $\Psi(x)=\theta$, then $x=\theta$; then there exists a bounded connected open set containing $\theta$ and such that there is at most one nonzero term in $\sum_{(d)}[\Phi, \omega, d][\Psi, d, \theta]$.

Proof. Let $\omega$ be a spherical neighborhood of $\theta$ such that $\omega$ is contained in $\Phi^{-1}(U)$. Now consider $\mathfrak{X}-\Phi\left(\omega^{\prime}\right)$. Since $\Phi\left(\omega^{\prime}\right)$ is closed, $\mathfrak{X}-\Phi\left(\omega^{\prime}\right)$ is an open set. This set has disjoint components $D_{1}, D_{2}, D_{3}, \cdots, D_{n}, \cdots$ which are also open since Banach space $\mathfrak{X}$ is locally connected. We consider the set of those $D_{i}$ such that $\left[\Phi, \omega, D_{i}\right] \neq 0$ and call this set $\left\{D_{i}\right\}$. Each such $D_{i}$ is contained in $\Phi(\omega)$ from the properties of topological degree. Since $U_{i} D_{i} \subset \Phi(\omega)$ $\subset U(\theta)$, it follows from the definition of $U(\theta)$ that only $D_{i}$ such that $\theta$ is an element of $D_{i}$ can be mapped so that $\Psi\left(D_{i}\right) \supset \theta$. Hence, at most one of the terms in $\sum_{(d)}[\Phi, \omega, d] \cdot[\Psi, d, \theta]$ is nonzero because at most one of the domains $D_{i}$ contains $\theta$. This completes the proof of the lemma.

Theorem 5.1. Given a mapping $I-C+T$ of Banach space $\mathfrak{X}$ into itself such that the Leray-Schauder topological degree is defined for $I-C+T$ and such that the multiplicity of Definition 3.1 is defined for $I-C+T$. If transformation $T$ satisfies $A$ ssumption 3.4 or Assumptions 3.5 and 3.6 of $\$ 3$, then $L(I-C+T)$ $=M(I-C+T)$ or $L(I-C+T)=(-1)[M(I-C+T)]$ where $L(I-C+T)$ is the Leray-Schauder topological degree at $\theta$ of $I-C+T$ relative to a sufficiently small sphere $\omega$ with center $\theta$, and $M(I-C+T)$ is the multiplicity of Definition 3.1 relative to the same point and sphere. 
Proof. By Theorem 2.8, $R=I+Q$ where $I$ is the identity transformation and $Q$ is linear, completely continuous. Moreover, $R$ is $1-1$. Hence $R$ has Leray-Schauder topological degree +1 or -1 relative to $\theta$ and $\omega$ (see $[10$, pp. 56-59)]. Hence by Lemmas 5.1 and 5.2, if $\omega$ is made sufficiently small,

$$
L[R(I-C+T)]=(L[R])(L[I-C+T])=( \pm 1) L[I-C+T] .
$$

We shall show that

$$
L[R(I-C+T)]=M[I-C+T] .
$$

This will complete the proof of the theorem.

We consider the equation

$$
R(I-C+T) x=R(y)
$$

which is equivalent to the equation $(I-C+T) x=y$ because there exists $R^{-1}$ (see Theorem $2.8, \S 2$ ). We carry out essentially the same procedure as used in $\S 3$. Denoting $R(y)$ by $z$ and applying Theorem 2.9, we obtain

$$
x-E_{1}(x)+R T(x)=z .
$$

Applying the transformations $E_{1}, E^{1}$ of Theorem 2.6 to equation (2), we obtain

$$
E_{1} R T[x]=E_{1} z \quad \text { or } \quad E_{1} R T\left[x_{1}+x^{1}\right]=E_{1} z=z_{1}
$$

and

$$
x^{1}+E^{1} R T\left[x_{1}+x^{1}\right]=E^{1} z=z^{1} .
$$

By Lemma 3.1, Theorem 2.10 can be applied to (4) and we obtain

$$
x^{1}=F\left(x_{1}, z^{1}\right) \text {. }
$$

Substituting from (5) into (3), we obtain

$$
E_{1} R T\left[x_{1}+F\left(x_{1}, z^{1}\right)\right]=E_{1} z=z_{1} \text {. }
$$

Now we defined two mappings $\Phi$ and $\Psi$ whose product is $R(I-C+T)$. The mapping $\Phi$ is defined as

$$
\Phi(x)=\Phi\left(x_{1}+x^{1}\right)=x_{1}+x^{1}+E^{1} R T\left[x_{1}+x^{1}\right]=x_{1}+z^{1} .
$$

The mapping $\Psi$ is defined as

$$
\begin{aligned}
\Psi\left(x_{1}+z^{1}\right)= & \left(I-E_{1}\right)\left(x_{1}+z^{1}\right) \\
& +E_{1} R T\left\{E_{1}\left(x_{1}+z^{1}\right)+F\left[E_{1}\left(x_{1}+z^{1}\right), E_{1}\left(x_{1}+z^{1}\right)\right]\right\} \\
= & z^{1}+E_{1} R T\left[x_{1}+F\left(x_{1}, z^{1}\right)\right] \\
= & z^{1}+z_{1}=z .
\end{aligned}
$$

It is clear from the definitions of $\Psi$ and $\Phi$ and from equations (1) and (2) that 
$\Psi \Phi=R(I-C+T)$.

Lemma 5.3. There exists a topological index (in the sense of Leray and Schauder) of $\Phi$ relative to $\theta$ and this topological index is +1 .

Proof. It is trivial to verify that the identity mapping $I$ is the differential (according to the definition of $[5$, p. 136]) at $\theta$ of the mapping $\Phi$ because of the $k$ th order properties of $T(k \geqq 2)$. The identity $I$ has topological index +1 (see $[10$, p. 55]). Now we apply Lemma $3, \S 1$ of [14]. By this lemma, the mapping $\Phi$ has topological index +1 .

Lemma 5.4. The Leray-Schauder topological degree at $\theta$ of the mapping $\Psi$ relative to a sufficiently small sphere $\omega$ with center $\theta$ is equal to the topological degree at $\theta$ of the mapping $E_{1} R T\left[x_{1}+F\left(x_{1}, z^{1}\right)\right]$ relative to the intersection of $\omega$ with $\mathfrak{X}_{1}$.

Proof. In the mapping $\Psi$, the mapping $E_{1} R T\left[x_{1}+F\left(x_{1}, z^{1}\right)\right]$ is a finitedimensional mapping into $\mathfrak{X}_{1}$. This mapping is continuous; also it is bounded. Thus $\Psi$ is a layer mapping according to the definition of $[15$, p. 374]. Hence by the definition of Leray-Schauder the topological degree at $\theta$ of $\Psi$ relative to a sphere with center $\theta$, is the topological degree of $\Psi$ regarded as a mapping of $\mathfrak{X}_{1}$ into $\mathfrak{X}_{1}$. If we denote this mapping by $\Psi / \mathfrak{X}_{1}$, it is clear from the definition of $\Psi$ that

$$
\Psi / \mathfrak{X}_{1}=E_{1} R T\left[x_{1}+F\left(x_{1}, z^{1}\right)\right] .
$$

This completes the proof of the lemma.

It is clear that Lemma 5.1 may be applied to these mappings $\Phi$ and $\Psi$. Moreover, since $\Phi(\theta)=\theta$ from the definition of $\Phi$, and since $\theta$ is an isolated $\theta$-point of $\Psi$ if Assumption 3.4 holds or if Assumptions 3.5 and 3.6 hold, the hypotheses of Lemma 5.2 are satisfied. Hence if we consider a sufficiently small sphere $\omega$ with $\theta$ as its center, then for topological degree $L$ and multiplicity $M$ relative to $\omega$ and $\theta$, we obtain

$$
L[R(I-C+T)]-L[\Psi \Phi]=\{L[\Psi]\}\{L[\Phi]\}=\{M(I-C+T)\}\{+1\} .
$$

This completes the proof of the theorem.

\section{BIBLIOGRAPHY}

1. P. Alexandroff and H. Hopf, Topologie I, Berlin, 1935. (Reprinted by Edwards Brothers, Ann Arbor, Mich.)

2. S. Banach, Theorie des operations lineaires, Warsaw, 1932.

3. R. Caccioppoli, Sulle corrispondenze funzionali inverse diramate: teoria generale e applicazione ad alcume equazioni funzionali non lineairi e al problema di Plateau, Rendiconti della Accademia Nazionale dei Lincei vol. 24 (1936) pp. 258-263.

4. T. H. Hildebrandt, Über vollstetige lineare Transformationen, Acta Math. vol. 51 (1928) pp. 311-318.

5. T. H. Hildebrandt and L. M. Graves, Implicit functions and their differentials in general analysis, Trans. Amer. Math. Soc. vol. 29 (1927) pp. 127-153. 
6. R. Iglisch, Reelle Lösungsfelder der elliptischen Differentialgleichung $\Delta u=F(u)$ und nichtlinearer Integralgleichungen, Math. Ann. vol. 101 (1927) pp. 98-119.

7. - Über die Vielfachheit einer Lösung in der Theorie der nichtlinearen Integralgleichungen von E. Schmidt, Jber. Deutschen Math. Verein. vol. 39 (1930) pp. 65-72.

8. - Zur Theorie der reellen Verzweigungen von Lösungen nichtlinearer Integralgleichungen, J. Reine Angew. Math. vol. 164 (1931) pp. 151-172.

9. J. Leray Note, Topologie des espaces abstrait de M. Banach, C. R. Acad. Sci. Paris vol. 200 (1935) pp. 1082-1084.

10. J. Leray, and J. Schauder, Topologie et equations fonctionnelles, Ann. École Norm. vol. 51 (1934) pp. 45-78.

11. A. Liapounoff, Sur les figures d'équilibre peu différentes des ellipsoïdes d'une masse liquide homogène douée d'un mouvement de rotation. Première Partie. Etude générale du problème, Mémoire presenté à l'Academie Imperiale des Sciences le 21 mars 1906, St. Petersbourg, pp. 1-225.

12. C. C. MacDuffee, Vectors and matrices, Carus Mathematical Monographs, No. 7, Mathematical Association of America, 1943.

13. F. Riesz, Über lineare Funktionalgleichungen, Acta Math. vol. 41 (1918) pp. 71-98.

14. E. H. Rothe, Topological proofs of uniqueness theorems in the theory of differential and integral equations, Bull. Amer. Math. Soc. vol. 45 (1939) pp. 606-613.

15. - The theory of topological order in some linear topological spaces, Iowa State College Journal of Science vol. 13 (1939) pp. 373-390.

16. J. Schauder, Über den Zusammenhang zwischen der Eindeutigkeit und Lösbarkeit partieller Differentialgleichungen zweiter Ordnung vom elliptischen Typus, Math. Ann. vol. 106 (1932) pp. 661-721.

17. E. Schmidt, Zur Theorie der linearen und nichtlinearen Integralgleichungen, Math. Ann. vol. 65 (1907-1908) pp. 370-399.

UNIVERSITY OF MichigAN, ANn ARbor, Mich. 